\title{
Effects of Traditional Chinese Medicine on Chemotherapy-Induced Myelosuppression and Febrile Neutropenia in Breast Cancer Patients
}

\author{
Huan Tian, ${ }^{1,2}$ Wei Qin, ${ }^{3}$ Wenjing Wu, ${ }^{1}$ Pi Guo, ${ }^{4}$ Yong Lu, ${ }^{4}$ Pengxi Liu, ${ }^{2}$ \\ Qiang Liu, ${ }^{1}$ and Fengxi Su${ }^{1}$ \\ ${ }^{1}$ Department of Breast Surgery, Sun Yat-sen Memorial Hospital, Sun Yat-sen University, 107 Yanjiangxi Road, \\ Guangzhou 510120, China \\ ${ }^{2}$ Department of Breast Surgery, Guangdong Hospital of Traditional Chinese Medicine, \\ Traditional Chinese Medicine University of Guangzhou, 111 Dade Road, Guangzhou 510120, China \\ ${ }^{3}$ Department of Hepatic Surgery, The Third Affiliated Hospital, Sun Yat-sen University, 600 Tianhe Road, Guangzhou 510630, China \\ ${ }^{4}$ Department of Medical Statistics and Epidemiology, School of Public Health, Sun Yat-sen University, \\ 74 Zhongshaner Road, Guangzhou 510080, China
}

Correspondence should be addressed to Fengxi Su; fengxisu@vip.163.com

Received 14 March 2015; Revised 26 May 2015; Accepted 3 June 2015

Academic Editor: Richard Pietras

Copyright (C) 2015 Huan Tian et al. This is an open access article distributed under the Creative Commons Attribution License, which permits unrestricted use, distribution, and reproduction in any medium, provided the original work is properly cited.

\begin{abstract}
Title. Chemotherapy-induced myelosuppression lowers the quality of life in breast cancer patients and causes many complications. Traditional Chinese Medicine (TCM) is a widely used complementary and alternative medicine therapies. Objective. To study whether TCM can reduce the incidence of chemotherapy-induced leukopenia, neutropenia, and febrile neutropenia (FN) in breast cancer patients. Methods. The data were analyzed retrospectively between patients who received TCM treatment (group 1,n=453) and patients who did not receive TCM treatment (group 2, $n=359$ ). Significant risk factors associated with the occurrence of chemotherapy-induced leukopenia, neutropenia, and FN were identified using multivariate analysis. Propensity score-matched patients were analyzed to adjust for any baseline differences. Results. Group 1 patients had a significantly lower rate of chemotherapyinduced severe leukopenia, neutropenia, and FN, compared with group $2(43 \%$ versus $71 \%, P<0.0001,72 \%$ versus $78 \%, P=0.005$, $6 \%$ versus $24 \%, P<0.0001$, resp.). Multivariate analysis revealed that chemotherapy regimens containing anthracyclines combined with paclitaxel or docetaxel were the most significant predictor. Subgroup analysis indicated that TCM treatment showed benefit in relieving chemotherapy-induced leukopenia and FN in most chemotherapy regimens. Conclusions. TCM treatment could lower the risk of severe chemotherapy-induced leukopenia, neutropenia, and FN in breast cancer patients.
\end{abstract}

\section{Introduction}

Chemotherapy is one of the major categories of pharmacotherapy for breast cancer. Chemotherapy-induced leukopenia, neutropenia, and FN are the most common and doselimiting toxicity of cytotoxic anticancer agents and often increase the susceptibility to the development of infections in breast cancer patients [1,2]. Moreover, severe leukopenia, neutropenia, and FN often result in treatment delay or dose reduction and discontinuation, which ultimately may compromise the long-term clinical outcome and lower the disease-free and overall survival rates [3-5].

TCM has a long history of application to disease treatment in China and has been used in numerous patients [6]. Some herbal components have also demonstrated antitumor activity by improving immune function [7]. Previous studies have demonstrated that Oldenlandia diffusa or Scutellaria barbata have antiproliferative effects on breast cancer cells in vitro and promising clinical effects in patients $[8,9]$. However, there is no report regarding the benefit of TCM in alleviating 
hematotoxicity. The aim of our study is to determine whether TCM can reduce the incidence of chemotherapy-induced leukopenia, neutropenia, and FN in breast cancer patients.

\section{Materials and Methods}

This study was approved by the Ethics Committees of Sun Yat-sen Memorial Hospital and Guangdong Hospital of Traditional Chinese Medicine, China. It was conducted in accordance with the Declaration of Helsinki.

2.1. Patient Population. This study was carried out on medical records of patients diagnosed with breast cancer who had been included, between January 2011 and April 2014, in prospective databases at Sun Yat-sen Memorial Hospital and Guangdong Hospital of Traditional Chinese Medicine, China. During the study period, 507 breast cancer patients from Guangdong Hospital of Traditional Chinese Medicine, and 521 from Sun Yat-sen Memorial Hospital were enrolled in the databases. Complete data were available from only 453 breast cancer patients in Guangdong Hospital of Traditional Chinese Medicine and 359 breast cancer patients in Sun Yat-sen Memorial Hospital. Breast cancer diagnosis was confirmed by needle biopsy or surgery.

2.2. Entry and Exclusion Criteria. Only patients who fulfill the following criteria were included in the retrospective analysis: age over 18 years, Chinese ethnic origin, life expectancy over 6 months, histological diagnosis of invasive breast cancer by core needle biopsy or surgery, requirement for chemotherapy according to the National Comprehensive Cancer Network (NCCN), an ECOG performance status of $0-1$, and the absence of fever for more than $24 \mathrm{hrs}$ before the start of chemotherapy. Pregnant women, patients with second primary malignant carcinomas or who had previously received chemotherapy or radiotherapy were excluded.

2.3. Treatment and Follow-Up. The enrolled patients were treated with chemotherapy according to NCCN. Before or during chemotherapy cycles, the white blood cells and absolute neutrophil count of these were recorded in succession. When diagnosed with chemotherapy-induced myelosuppression (leukopenia or neutropenia), patients in Guangdong Hospital of Traditional Chinese Medicine (group 1), received TCM decoctions for jian pi (to regulate the gastrointestinal function for better assimilation) and yi qi yang xue sheng sui (to enhance the hematopoietic activity of the bone marrow), while the patients in Sun Yat-sen Memorial Hospital (group 2) did not.

When severe myelosuppression occurred (i.e., leukocyte lower than $2.0 \times 10^{9} / \mathrm{L}$ or neutrophils lower than $1.0 \times 10^{9} / \mathrm{L}$ ), group 1 patients received the treatment of granulocyte colonystimulating factor (G-CSF), in addition to TCM decoctions. In group 2, these patients received only G-CSF treatment. Meanwhile, body temperatures of breast cancer patients should be recorded. FN (i.e., body temperature $\geq 38.2^{\circ} \mathrm{C}$ and absolute neutrophil count $<0.5 \times 10^{9} / \mathrm{L}$ on the same day of the fever or the day after) was defined according to the definition used by the Infectious Disease Society of America (IDSA) and the European Organization for Research and Treatment of Cancer (EORTC) [10, 11].

The data of alanine transaminase (ALT), aspartate aminotransferase (AST), blood urea nitrogen (BUN), and creatinine (CR) were recorded in every chemotherapy cycle to evaluate the toxicity of TCM.

2.4. Statistical Analysis. All demographic and clinicopathological data had been prospectively collected in computer databases before this retrospective analysis. Continuous variables were expressed as the mean. Categorical variables were reported as the number and percentage. Differences between continuous data were analyzed using Mann-Whitney test. Differences between categorical data were analyzed using the $\chi^{2}$ test. Significant risk factors associated with the occurrence of chemotherapy-induced leukopenia, neutropenia, and FN were identified using logistic regression model multivariate analysis. Correlation power of all the risk factors was evaluated by the odds ratio (OR). SPSS 17.0 statistical package (SPSS, Inc., Chicago, IL, USA) was used for the statistical analyses.

To reduce bias in patient selection, propensity analysis was carried out using logistic regression to create propensity scores for the treatment and control patients. Logistic regression was applied to the studied clinical variables differing significantly between the treatment and control patients with chemotherapy-induced myelosuppression and FN, and propensity scores were generated along a continuous range from 0 to 1 . The model was then used to provide a oneto-one nearest-neighbor match between patients undergoing treatment or control. The propensity analysis was established using the matching package within $\mathrm{R}$ 3.0.2 software which was used for the statistical analyses [12].

All reported $P$ values were those of two-sided tests. The statistical significance was set at $P<0.05$.

\section{Results}

3.1. Clinical and Demographic Characteristics of the Breast Cancer Patients. On the basis of the inclusion criteria, 812 breast cancer patients were enrolled in this retrospective study. Of these patients, 453 received TCM treatment and 359 underwent no TCM treatment (Figure 1). Baseline demographic and clinicopathological data were summarized in Table 1. There were significant differences in age $(P=$ $0.000004)$, chemotherapy regimens $(P<0.0001)$, receiving preoperative chemotherapy $(P=0.0029)$, status of estrogen receptor $(\mathrm{ER})(P=0.000149)$, progesterone receptor $(\mathrm{PR})$ $(P=0.000244)$, human epidermal growth factor receptor$2(\operatorname{HER}-2)(P=0.003203)$, and $\operatorname{Ki67}(P<0.0001)$ between the two groups. There was no significant difference in tumor stage $(P=0.4594)$. Propensity analysis based on variables associated with the therapeutic strategy and myelosuppression identified 577 matched pairs of patients from each group. When only these pairs were considered, the two groups did not exhibit significant baseline difference in age, chemotherapy regimens, tumor stage, receiving preoperative 


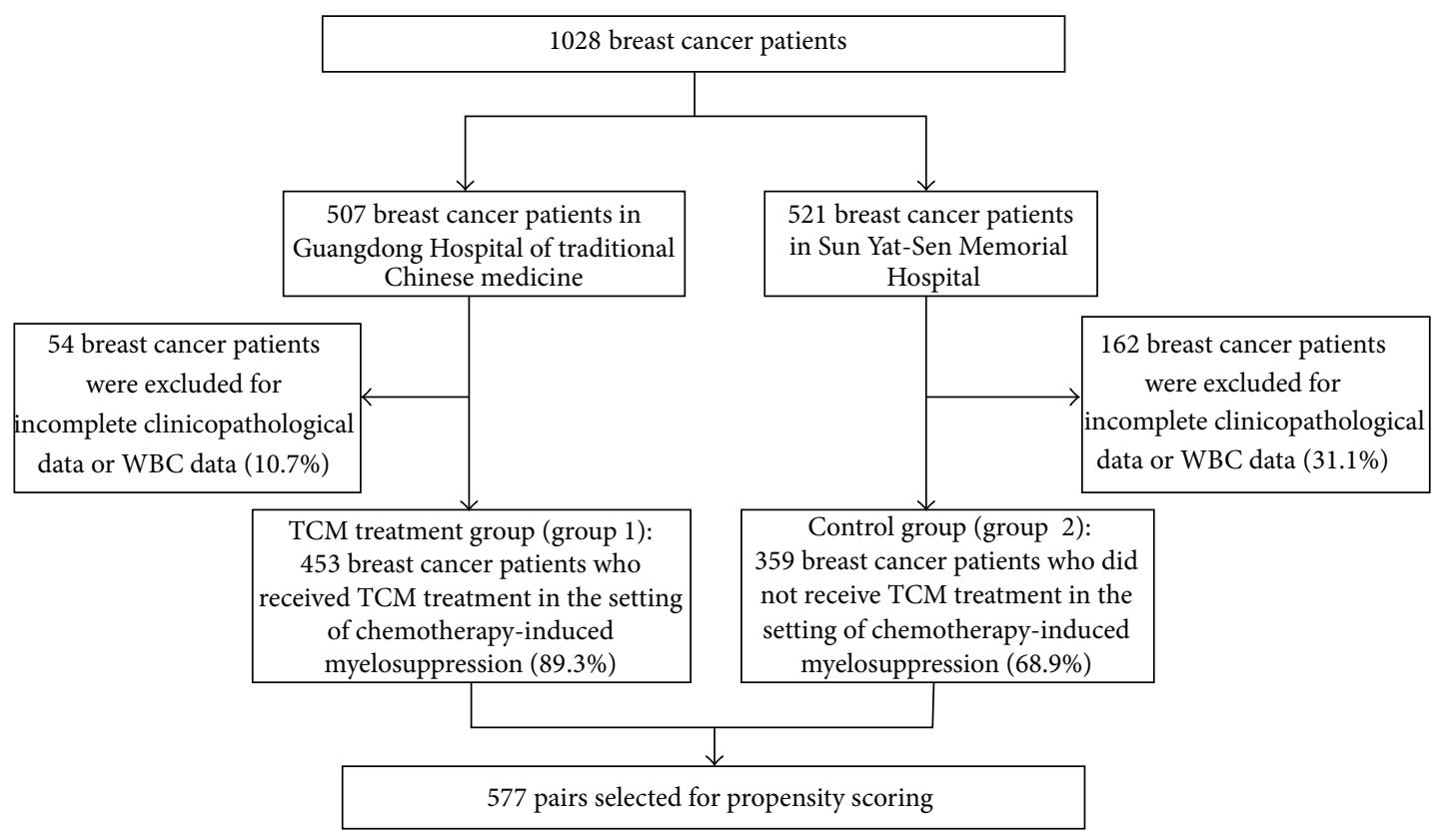

Figure 1: Flow diagram of the study. The patients databases included 1028 breast cancer patients. In TCM treatment group (group 1), 453 breast cancer patients received TCM treatment in the setting of chemotherapy-induced myelosuppression. In control group (group 2), 359 breast cancer patients did not receive TCM treatment in the setting of chemotherapy-induced myelosuppression. In the propensity score model, 577 pairs of matched patients were generated for baseline-adjusted analyses.

chemotherapy, and status of ER, PR, HER-2, Ki67, and AST (Table 1).

\subsection{TCM Decreased the Rate of Chemotherapy-Induced} Leukopenia, Neutropenia, and FN in the Entire Study Population. When receiving chemotherapy, there were significant differences between the two groups with respect to chemotherapy-induced leukopenia, neutropenia, and FN (Table 2). Patients in group 1 had a significantly lower rate of severe leukopenia and neutropenia compared with the patients in group 2 ( $43 \%$ versus $71 \%, P<0.0001 ; 72 \%$ versus $79 \%, P=0.005$, resp.) (Figure 2). Furthermore, patients selected in the propensity-matching model who underwent TCM treatment exhibited a significantly lower rate of FN compared with those who did not undergo TCM treatment (6\% versus $24 \%, P<0.0001$ ) (Figure 2 ).

\subsection{Multivariate Analysis of Clinicopathological Factors Pre-} dictive of Chemotherapy-Induced Leukopenia, Neutropenia, and FN. Significant risk factors associated with the occurrence of chemotherapy-induced leukopenia, neutropenia, and FN were identified using logistic regression analysis. The variables in the multivariate model included age, chemotherapy chemotherapy regimens, tumor stage, pathologic features, and other factors (Tables 3-5).

The multivariate analysis revealed that chemotherapy regimens containing anthracyclines combined with paclitaxel or docetaxel associated with a significant risk of chemotherapy-induced leukopenia (OR 3.208, 95\% CI 2.228-4.617,
$P<0.0001$ ), neutropenia (OR 4.184, 95\% CI 2.725-6.424, $P<0.0001$ ), and FN (OR 4.304, 95\% CI 2.641-7.015, $P<$ 0.0001 ) in the total population. The tumor stage associated with a significant risk of leukopenia (stage II, OR 1.360, 95\% CI 1.014-1.823, $P=0.04$; stages III and IV, OR 1.953, 95\% CI 1.215-3.141, $P=0.006$ ) and neutropenia (stage II, OR 1.554, $95 \%$ CI 1.139-2.120, $P=0.005$ ) but not the risk of FN. The Ki67 index could increase the risk of leukopenia (OR 1.552, 95\% CI 1.163-2.071, $P=0.003$ ) but not the risk of neutropenia and FN. PR could increase the risk of FN (OR 2.631, 95\% CI $1.418-4.882, P=0.002)$ but not the risk of leukopenia and neutropenia.

Factor protected against chemotherapy-induced leukopenia, neutropenia, and FN was TCM treatment. In the propensity-matching model, the OR of leukopenia, neutropenia, and FN for the TCM treatment were 0.285 (95\% CI 0.218 $0.373, P<0.0001), 0.741$ (95\% CI 0.554-0.992, $P=0.044$ ), and 0.184 (95\% CI, $0.122-0.279, P<0.0001$ ), respectively.

The factors, such as younger age and not receiving preoperative chemotherapy, could decrease the risk of leukopenia but not the risk of neutropenia and FN. The OR were 0.979 (95\% CI, 0.965-0.994, $P=0.005$ ) and 0.584 (95\% CI, 0.377$0.905, P=0.016)$, respectively.

No other characteristics were associated with increased risk of leukopenia, neutropenia, and FN: ER and HER-2 $(P>$ 0.05).

3.4. Subgroup Analysis of the Entire Study Population. To explore more deeply the efficacy of chemotherapy regimens 
TABLE 1: Clinicopathological data of breast cancer patients.

\begin{tabular}{|c|c|c|c|c|c|c|}
\hline \multirow{2}{*}{ Variables } & \multicolumn{3}{|c|}{ Before propensity matching } & \multicolumn{3}{|c|}{ After propensity matching } \\
\hline & Group $1(n=453)$ & Group $2(n=359)$ & $P$ & Group $1(n=577)$ & Group $2(n=577)$ & $P$ \\
\hline Age, yr & $50.3(49.4-51.2)$ & $47.1(46.1-48.1)$ & 0.000004 & $49.8(49.0-50.6)$ & $50.2(49.3-51.0)$ & 0.504 \\
\hline Chemotherapy & & & $<0.0001$ & & & 0.250 \\
\hline 1 & 154 & 35 & & 179 & 173 & \\
\hline 2 & 42 & 54 & & 63 & 52 & \\
\hline 3 & 172 & 183 & & 229 & 260 & \\
\hline 4 & 85 & 87 & & 106 & 92 & \\
\hline TNM stage & & & 0.4594 & & & 0.200 \\
\hline I & 164 & 117 & & 215 & 164 & \\
\hline II & 219 & 189 & & 275 & 333 & \\
\hline III and IV & 70 & 53 & & 87 & 80 & \\
\hline Neoadjuvant chemotherapy & & & 0.0029 & & & 0.637 \\
\hline Yes & 73 & 88 & & 94 & 100 & \\
\hline No & 380 & 271 & & 483 & 477 & \\
\hline ER & & & 0.000149 & & & 0.899 \\
\hline- & 146 & 73 & & 179 & 177 & \\
\hline+ & 307 & 286 & & 398 & 400 & \\
\hline PR & & & 0.000244 & & & 0.811 \\
\hline- & 193 & 108 & & 237 & 241 & \\
\hline+ & 260 & 251 & & 340 & 336 & \\
\hline HER-2 & & & 0.003203 & & & 0.052 \\
\hline- & 340 & 300 & & 441 & 468 & \\
\hline+ & 113 & 59 & & 136 & 109 & \\
\hline Ki67 & & & $<0.0001$ & & & 0.813 \\
\hline- & 256 & 93 & & 304 & 308 & \\
\hline+ & 197 & 266 & & 273 & 269 & \\
\hline $\operatorname{ALT}(\mathrm{U} / \mathrm{L})$ & & & 0.000038 & & & 0.008 \\
\hline$\leq 40$ & 382 & 257 & & 487 & 446 & \\
\hline$>40$ & 71 & 102 & & 90 & 131 & \\
\hline AST (U/L) & & & 0.004 & & & 0.224 \\
\hline$\leq 40$ & 394 & 281 & & 499 & 483 & \\
\hline$>40$ & 59 & 78 & & 78 & 94 & \\
\hline
\end{tabular}

HER-2 positive: testing by immunohistochemical (IHC) assay (3+) or in situ hybridization (ISH) assay (+); HER-2 negative: IHC (-), (1+), and (2+) or ISH (-); ALT: alanine aminotransferase; AST: aspartate transferase. Chemotherapy: 1: the chemotherapy regimens contain AC/EC (Adriamycin or epirubicin, Cyclophosphamide) and CAF/CEF (Adriamycin or epirubicin, Cyclophosphamide, and 5-Fluorouracil). 2: the chemotherapy regimens contain TC (paclitaxel or docetaxel, Cyclophosphamide) and $\mathrm{T}$ (paclitaxel or docetaxel). 3: the chemotherapy regimens contain anthracyclines combined with paclitaxel or docetaxel: TAC/TEC (paclitaxel or docetaxel, Cyclophosphamide, and Adriamycin or epirubicin); TA/TE (paclitaxel or docetaxel, Adriamycin or epirubicin). 4: the chemotherapy regimens contain anthracyclines followed by paclitaxel or docetaxel: AC/EC (Adriamycin or epirubicin, Cyclophosphamide); CAF/CEF (Adriamycin or epirubicin, Cyclophosphamide, and 5-Fluorouracil), followed with T or TH (paclitaxel or docetaxel, Herceptin).

and tumor stage for chemotherapy-induced leukopenia, neutropenia, and FN, we performed subgroup analysis.

3.5. Subgroup Analysis by Chemotherapy Regimens. Patients in each group were divided into subgroups with different chemotherapy regimens. TCM treatment showed a benefit in relieving chemotherapy-induced leukopenia and $\mathrm{FN}$ in most chemotherapy regimens but little benefit in chemotherapy-induced neutropenia (see Supporting Tables 1-3 in Supplementary Material available online at http://dx.doi.org/ 10.1155/2015/736197).

Among patients with regimens containing paclitaxel or docetaxel, TCM provided lower rate of severe leukopenia
(2\% versus $5 \%, P=0.000006$ ), neutropenia (3\% versus $6 \%$, $P=0.000012)$, and $\mathrm{FN}(0 \%$ versus $1 \%, P=0.003053)$ than control group (Figures 3-5). Among patients with regimens containing anthracyclines only and regimens containing anthracyclines followed by paclitaxel or docetaxel, TCM provided lower severe leukopenia (6\% versus $20 \%, P<$ $0.0001 ; 7 \%$ versus $11 \%, P=0.000034$, resp.) and $\mathrm{FN}(0 \%$ versus $5 \%, P<0.0001 ; 0 \%$ versus $4 \%, P<0.0001$, resp.), but there was no significant difference in severe neutropenia (19\% versus $20 \%, P=0.235 ; 15 \%$ versus $12 \%, P=0.485$, resp.) (Figures 3-5). Among patients with regimens containing anthracyclines combined with paclitaxel or docetaxel, TCM provided lower rate of $\mathrm{FN}$ ( $5 \%$ versus $14 \%, P=0.000001$ ), 
TABLE 2: Different outcomes in chemotherapy-induced leukopenia, neutropenia, and FN between two groups.

\begin{tabular}{|c|c|c|c|c|c|c|}
\hline \multirow{2}{*}{ Outcomes } & \multicolumn{3}{|c|}{ Before propensity matching } & \multicolumn{3}{|c|}{ After propensity matching } \\
\hline & Group $1(n=453)$ & Group $2(n=359)$ & $P$ & Group $1(n=577)$ & Group $2(n=577)$ & $P$ \\
\hline Leukopenia & & & $<0.0001$ & & & $<0.0001$ \\
\hline Mild & 261 & 85 & & 328 & 169 & \\
\hline Severe & 192 & 274 & & 249 & 408 & \\
\hline Neutropenia & & & 0.000842 & & & 0.005 \\
\hline Mild & 126 & 64 & & 163 & 122 & \\
\hline Severe & 327 & 295 & & 414 & 455 & \\
\hline Febrile neutropenia & & & $<0.0001$ & & & $<0.0001$ \\
\hline No & 428 & 254 & & 545 & 439 & \\
\hline Yes & 25 & 105 & & 32 & 138 & \\
\hline
\end{tabular}

Mild: Grades I and II myelosuppression. Leukocyte lower than normal but higher than (or equal to) $2.0 \times 10^{9} / \mathrm{L}$; neutrophils lower than normal but higher than (or equal to) $1.0 \times 10^{9} / \mathrm{L}$. Severe: Grades III and IV myelosuppression. Leukocyte lower than $2.0 \times 10^{9} / \mathrm{L}$; neutrophils lower than $1.0 \times 10^{9} / \mathrm{L}$. FN: body temperature $\geq 38.2^{\circ} \mathrm{C}$ and absolute neutrophil count $<0.5 \times 10^{9} / \mathrm{L}$ on the same day of the fever or the day after.

TABLE 3: Multivariate analysis of clinicopathological variables of leukopenia.

\begin{tabular}{|c|c|c|c|c|c|c|}
\hline \multirow{2}{*}{ Variables } & \multicolumn{3}{|c|}{ Before propensity matching } & \multicolumn{3}{|c|}{ After propensity matching } \\
\hline & OR & $95 \% \mathrm{CI}$ & $P$ & OR & $95 \% \mathrm{CI}$ & $P$ \\
\hline Age & 0.989 & $0.971-1.006$ & 0.199 & 0.979 & $0.965-0.994$ & 0.005 \\
\hline \multicolumn{7}{|l|}{ Chemotherapy regimens } \\
\hline 1 & 1 & - & - & 1 & - & - \\
\hline 2 & 0.976 & $0.539-1.765$ & 0.935 & 0.773 & $0.468-1.274$ & 0.312 \\
\hline 3 & 4.477 & $2.764-7.251$ & $<0.0001$ & 3.208 & $2.228-4.617$ & $<0.0001$ \\
\hline 4 & 1.552 & $0.942-2.558$ & 0.085 & 1.251 & $0.837-1.869$ & 0.275 \\
\hline \multicolumn{7}{|l|}{ TNM stage } \\
\hline I & 1 & - & - & 1 & - & - \\
\hline II & 1.227 & $0.858-1.756$ & 0.262 & 1.360 & $1.014-1.823$ & 0.040 \\
\hline III and IV & 2.436 & $1.362-4.357$ & 0.003 & 1.953 & $1.215-3.141$ & 0.006 \\
\hline Neoadjuvant chemotherapy & 0.788 & $0.476-1.304$ & 0.354 & 0.584 & $0.377-0.905$ & 0.016 \\
\hline ER & 1.165 & $0.655-2.071$ & 0.603 & 1.215 & $0.772-1.913$ & 0.399 \\
\hline PR & 0.781 & $0.457-1.333$ & 0.364 & 1.003 & $0.654-1.537$ & 0.989 \\
\hline HER-2 & 0.857 & $0.561-1.307$ & 0.473 & 1.094 & $0.762-1.572$ & 0.626 \\
\hline Ki67 & 1.132 & $0.791-1.620$ & 0.499 & 1.552 & $1.163-2.071$ & 0.003 \\
\hline TCM treatment & 0.252 & $0.172-0.367$ & $<0.0001$ & 0.285 & $0.218-0.373$ & $<0.0001$ \\
\hline
\end{tabular}

Chemotherapy: 1: the chemotherapy regimens contain AC/EC (Adriamycin or epirubicin, Cyclophosphamide) and CAF/CEF (Adriamycin or epirubicin, Cyclophosphamide, and 5-Fluorouracil). 2: the chemotherapy regimens contain TC (paclitaxel or docetaxel, Cyclophosphamide) and T (paclitaxel or docetaxel). 3: the chemotherapy regimens contain anthracyclines combined with paclitaxel or docetaxel: TAC/TEC (paclitaxel or docetaxel, Cyclophosphamide, and Adriamycin or epirubicin); TA/TE (paclitaxel or docetaxel, Adriamycin or epirubicin). 4: the chemotherapy regimens contain anthracyclines followed by paclitaxel or docetaxel: AC/EC (Adriamycin or epirubicin, Cyclophosphamide); CAF/CEF (Adriamycin or epirubicin, Cyclophosphamide, and 5-Fluorouracil), followed with T or TH (paclitaxel or docetaxel, Herceptin).

but there was no significant difference in severe leukopenia (28\% versus $35 \%, P=0.08$ ) and neutropenia (35\% versus $41 \%, P=0.628$ ) (Figures 3-5).

3.6. Subgroup Analysis by Tumor Stage. Patients were divided into subgroups with tumor stage. Among patients at stage II, severe leukopenia ( $18 \%$ versus $43 \%, P<0.0001$ ), severe neutropenia (34\% versus $48 \%, P=0.000093)$, and $\mathrm{FN}(1 \%$ versus $15 \%, P<0.0001)$ were significantly lower in group 1 (Supporting Tables 4-6) (Figures 6-8). Among patients at stage I, severe leukopenia ( $13 \%$ versus $18 \%, P<0.0001$ ) and FN (3\% versus $6 \%, P=0.000152$ ) were significantly lower in group 1. But there was no significant difference in severe neutropenia ( $24 \%$ versus $19 \%, P=0.537$ ) between two groups (Supporting Tables 4-6) (Figures 6-8). Among patients at stage III, TCM treatment showed little effect in relieving chemotherapy-induced severe leukopenia (12\% versus $10 \%$, $P=0.227)$, severe neutropenia ( $14 \%$ versus $11 \%, P=0.021)$, and FN (2\% versus 3\%, $P=0.052$ ) (Supporting Tables 4-6) (Figures 6-8).

3.7. The Toxicity of TCM. There was no patient suffering from renal damage during the chemotherapy in TCM treatment group. Hepatic dysfunction could be found in both groups. 
TABLE 4: Multivariate analysis of clinicopathological variables of neutropenia.

\begin{tabular}{|c|c|c|c|c|c|c|}
\hline \multirow{2}{*}{ Variables } & \multicolumn{3}{|c|}{ Before propensity matching } & \multicolumn{3}{|c|}{ After propensity matching } \\
\hline & OR & $95 \% \mathrm{CI}$ & $P$ & OR & $95 \% \mathrm{CI}$ & $P$ \\
\hline Age & 1.006 & $0.987-1.026$ & 0.516 & 0.998 & $0.982-1.014$ & 0.777 \\
\hline \multicolumn{7}{|l|}{ Chemotherapy regimens } \\
\hline 1 & 1 & - & - & 1 & - & - \\
\hline 2 & 0.592 & $0.336-1.045$ & 0.071 & 0.580 & $0.361-0.931$ & 0.024 \\
\hline 3 & 3.853 & $2.245-6.614$ & 0.000001 & 4.184 & $2.725-6.424$ & $<0.0001$ \\
\hline 4 & 2.124 & $1.252-3.606$ & 0.005 & 1.984 & $1.282-3.072$ & 0.002 \\
\hline \multicolumn{7}{|l|}{ TNM stage } \\
\hline I & 1 & - & - & 1 & - & - \\
\hline II & 1.157 & $0.797-1.678$ & 0.443 & 1.554 & $1.139-2.120$ & 0.005 \\
\hline III and IV & 2.029 & $0.989-4.164$ & 0.054 & 1.683 & $0.952-2.967$ & 0.073 \\
\hline Neoadjuvant chemotherapy & 0.962 & $0.523-1.768$ & 0.901 & 0.896 & $0.521-1.541$ & 0.691 \\
\hline ER & 1.134 & $0.600-2.144$ & 0.698 & 0.932 & $0.556-1.561$ & 0.789 \\
\hline PR & 0.957 & $0.533-1.718$ & 0.883 & 0.969 & $0.602-1.560$ & 0.898 \\
\hline HER-2 & 0.962 & $0.599-1.546$ & 0.873 & 1.090 & $0.722-1.645$ & 0.682 \\
\hline Ki67 & 0.859 & $0.582-1.266$ & 0.442 & 1.022 & $0.745-1.402$ & 0.893 \\
\hline TCM treatment & 0.607 & $0.400-0.923$ & 0.019 & 0.741 & $0.554-0.992$ & 0.044 \\
\hline
\end{tabular}

Chemotherapy: 1: the chemotherapy regimens contain AC/EC (Adriamycin or epirubicin, Cyclophosphamide) and CAF/CEF (Adriamycin or epirubicin, Cyclophosphamide, and 5-Fluorouracil). 2: the chemotherapy regimens contain TC (paclitaxel or docetaxel, Cyclophosphamide) and T (paclitaxel or docetaxel). 3: the chemotherapy regimens contain anthracyclines combined with paclitaxel or docetaxel: TAC/TEC (paclitaxel or docetaxel, Cyclophosphamide, and Adriamycin or epirubicin); TA/TE (paclitaxel or docetaxel, Adriamycin or epirubicin). 4: the chemotherapy regimens contain anthracyclines followed by paclitaxel or docetaxel: AC/EC (Adriamycin or epirubicin, Cyclophosphamide); CAF/CEF (Adriamycin or epirubicin, Cyclophosphamide, and 5-Fluorouracil), followed with T or TH (paclitaxel or docetaxel, Herceptin).

TABLE 5: Multivariate analysis of clinicopathological variables of FN.

\begin{tabular}{|c|c|c|c|c|c|c|}
\hline \multirow{2}{*}{ Variables } & \multicolumn{3}{|c|}{ Before propensity matching } & \multicolumn{3}{|c|}{ After propensity matching } \\
\hline & OR & $95 \% \mathrm{CI}$ & $P$ & OR & $95 \% \mathrm{CI}$ & $P$ \\
\hline Age & 0.985 & $0.963-1.007$ & 0.182 & 0.995 & $0.979-1.014$ & 0.590 \\
\hline \multicolumn{7}{|l|}{ Chemotherapy regimens } \\
\hline 1 & 1 & - & - & 1 & - & - \\
\hline 2 & 1.797 & $0.585-5.514$ & 0.306 & 0.785 & $0.321-1.918$ & 0.595 \\
\hline 3 & 6.433 & $2.612-15.844$ & 0.000052 & 4.304 & $2.641-7.015$ & $<0.0001$ \\
\hline 4 & 2.516 & $0.942-6.721$ & 0.066 & 1.917 & $1.035-3.552$ & 0.039 \\
\hline \multicolumn{7}{|l|}{ TNM stage } \\
\hline $\mathrm{I}$ & 1 & - & - & 1 & - & - \\
\hline II & 0.895 & $0.542-1.478$ & 0.665 & 0.925 & $0.613-1.396$ & 0.711 \\
\hline III and IV & 0.897 & $0.457-1.757$ & 0.750 & 0.690 & $0.383-1.244$ & 0.217 \\
\hline Neoadjuvant chemotherapy & 0.710 & $0.426-1.184$ & 0.190 & 1.519 & $0.916-2.522$ & 0.106 \\
\hline ER & 0.588 & $0.289-1.195$ & 0.142 & 0.544 & $0.291-1.014$ & 0.055 \\
\hline PR & 1.404 & $0.720-2.738$ & 0.319 & 2.631 & $1.418-4.882$ & 0.002 \\
\hline HER-2 & 0.803 & $0.464-1.391$ & 0.434 & 1.009 & $0.622-1.637$ & 0.970 \\
\hline Ki67 & 0.946 & $0.589-1.520$ & 0.819 & 1.182 & $0.812-1.721$ & 0.383 \\
\hline TCM treatment & 0.166 & $0.098-0.280$ & $<0.0001$ & 0.184 & $0.122-0.279$ & $<0.0001$ \\
\hline
\end{tabular}

Chemotherapy: 1: the chemotherapy regimens contain AC/EC (Adriamycin or epirubicin, Cyclophosphamide) and CAF/CEF (Adriamycin or epirubicin, Cyclophosphamide, and 5-Fluorouracil). 2: the chemotherapy regimens contain TC (paclitaxel or docetaxel, Cyclophosphamide) and T (paclitaxel or docetaxel). 3: the chemotherapy regimens contain anthracyclines combined with paclitaxel or docetaxel: TAC/TEC (paclitaxel or docetaxel, Cyclophosphamide, and Adriamycin or epirubicin); TA/TE (paclitaxel or docetaxel, Adriamycin or epirubicin). 4: the chemotherapy regimens contain anthracyclines followed by paclitaxel or docetaxel: AC/EC (Adriamycin or epirubicin, Cyclophosphamide); CAF/CEF (Adriamycin or epirubicin, Cyclophosphamide, and 5-Fluorouracil), followed with $\mathrm{T}$ or TH (paclitaxel or docetaxel, Herceptin). FN: body temperature $\geq 38.2^{\circ} \mathrm{C}$ and absolute neutrophil count $<0.5 \times 10^{9} / \mathrm{L}$ on the same day of the fever or the day after. 


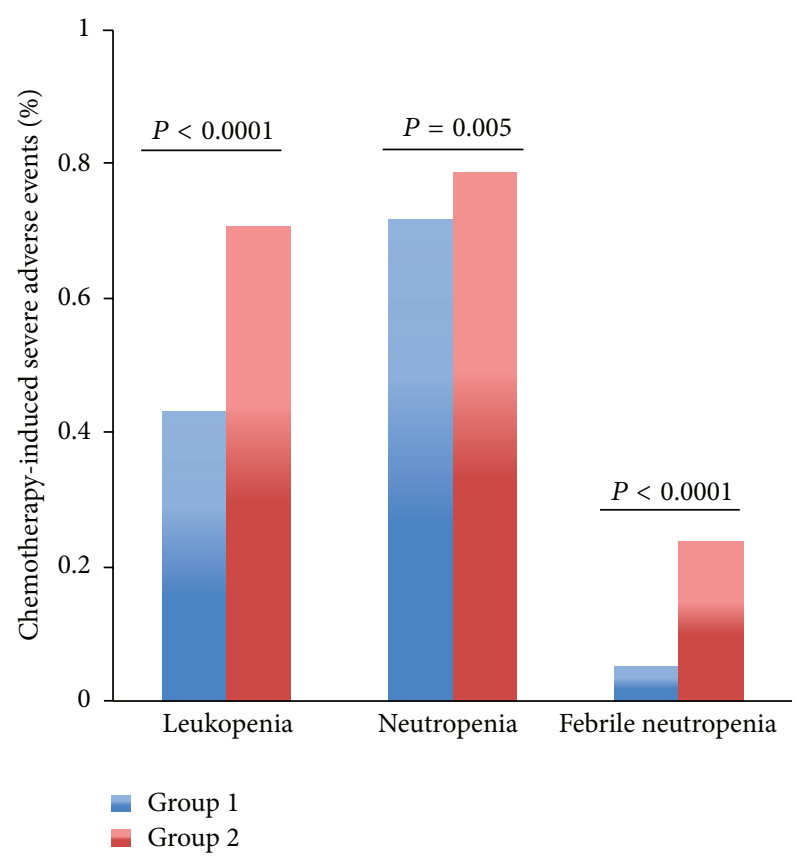

FIGURE 2: The rates of severe chemotherapy-induced leukopenia, neutropenia (Grades III-IV), and FN for the entire propensitymatched patients. After matching, patients in group 2 had a significantly higher rate of severe leukopenia, neutropenia, and FN compared to patients in group 1 (43\% versus 71\%, $P<0.0001 ; 72 \%$ versus $79 \%, P=0.005 ; 6 \%$ versus $24 \%, P<0.0001$, resp.).

The numbers of patients in group 1 and group 2 concomitant with ALT elevation were 71 and 102, respectively. However, the numbers of patients in group 1 and group 2 concomitant with AST elevation were 59 and 78, respectively.

After propensity matching, the rates of ALT elevation were $16 \%$ in group 1 and $23 \%$ in group $2(P=0.004167)$. The rates of AST elevation were $14 \%$ in group 1 and $16 \%$ in group $2(P=0.0224)$ (Supporting Table 7).

\section{Discussion}

Chemotherapy-induced myelosuppression is an important and often dose-limiting toxicity of cytotoxic anticancer agents. It may lower the quality of life in breast cancer patients and be associated with many complications, including increased risks for opportunistic infections, $\mathrm{FN}$, sepsis, and mortality.

TCM is considered as an appropriate therapy for managing chronic diseases such as cancer but mainly for symptom relief or palliative care rather than for curing the disease. Previous studies had shown that some TCMs could reduce the side effects of chemotherapy, modulate immune effector cells, and relieve chemotherapy-induced myelosuppression [13-16]. Researchers explained the effectiveness of some herbs on neutrophils by biochemical tests and then attributed these to $[17-20]$ (i) improving the bone marrow hematopoietic microenvironment; (ii) improving the cyclin D1 expression, promoting cell cycles in the G0/G1 phase to enter the S,

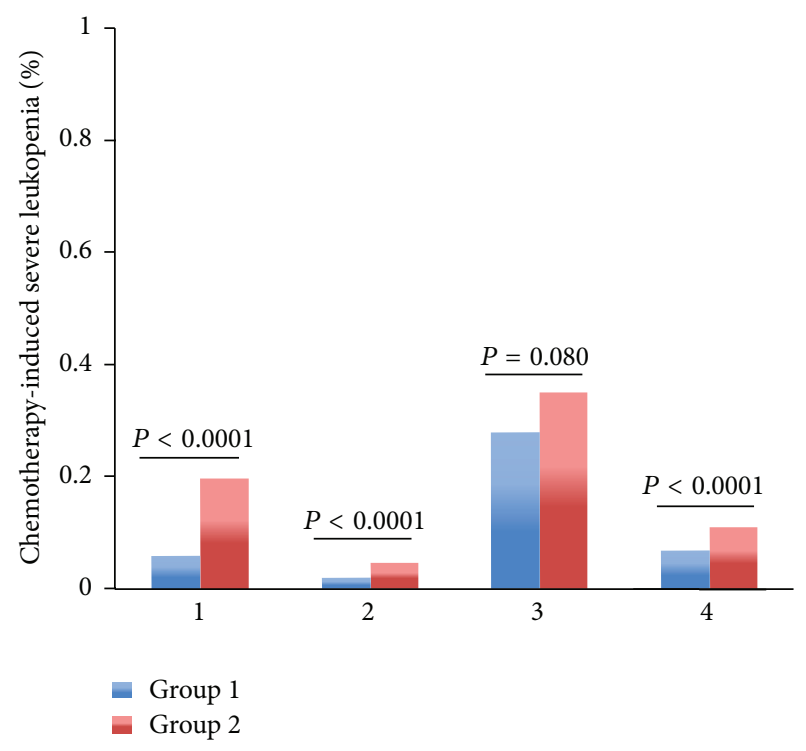

FIGURE 3: The rates of severe chemotherapy-induced leukopenia (Grades III-IV) for the entire propensity-matched patients in different chemotherapy regimens. After matching, patients in group 2 had a higher rate of severe leukopenia compared to patients in group 1 (20\% versus $6 \%, P<0.0001 ; 5 \%$ versus $2 \%, P<0.0001 ; 35 \%$ versus $28 \%, P=0.080 ; 11 \%$ versus $7 \%$, $P<0.0001$, resp.). Chemotherapy. 1: the chemotherapy regimens contain AC/EC (Adriamycin or epirubicin, Cyclophosphamide) and $\mathrm{CAF} / \mathrm{CEF}$ (Adriamycin or epirubicin, Cyclophosphamide, and 5Fluorouracil). 2: the chemotherapy regimens contain TC (paclitaxel or docetaxel, Cyclophosphamide) and $\mathrm{T}$ (paclitaxel or docetaxel). 3: the chemotherapy regimens contain anthracyclines combined with paclitaxel or docetaxel: TAC/TEC (paclitaxel or docetaxel, Cyclophosphamide, and Adriamycin or epirubicin); TA/TE (paclitaxel or docetaxel, Adriamycin or epirubicin). 4: the chemotherapy regimens contain anthracyclines followed by paclitaxel or docetaxel: AC/EC (Adriamycin or epirubicin, Cyclophosphamide); CAF/CEF (Adriamycin or epirubicin, Cyclophosphamide, and 5Fluorouracil), followed with $\mathrm{T}$ or $\mathrm{TH}$ (paclitaxel or docetaxel, Herceptin).

G2/M phases to accelerate hematopoietic progenitor cell proliferation and differentiation, and involving the PI3K/AKT pathway to thereby prevent bone marrow nucleated cells from apoptosis; and (iii) regulating the immune function and the expression of CDK4 and CDK6 in bone marrow and tumor tissues, stimulating the expression of IL- $1 \beta$, IL-3, IL-6, SCF, and GM-CSF and inhibiting the expression of TGF- $\beta$.

The clinical studies on the effects of TCM on chemotherapy-induced myelosuppression in breast cancer patients were little. Consequently, we proposed this research by treating myelosuppression patients with TCM and G-CSF compared with G-CSF alone. We demonstrated that TCM treatment provided an absolute risk reduction of severe leukopenia events by $28 \%(P<0.0001)$. Furthermore, TCM treatment also reduced the risk of severe neutropenia events by $7 \%$ $(P=0.005)$ and reduced the risk of FN event by $18 \%(P<$ $0.0001)$ in breast cancer patients. Previous studies had shown that the morbidity of FN in treatment-naive patients was approximately $15-40 \%$ [21, 22]. While, in our study, $24 \%$ of 


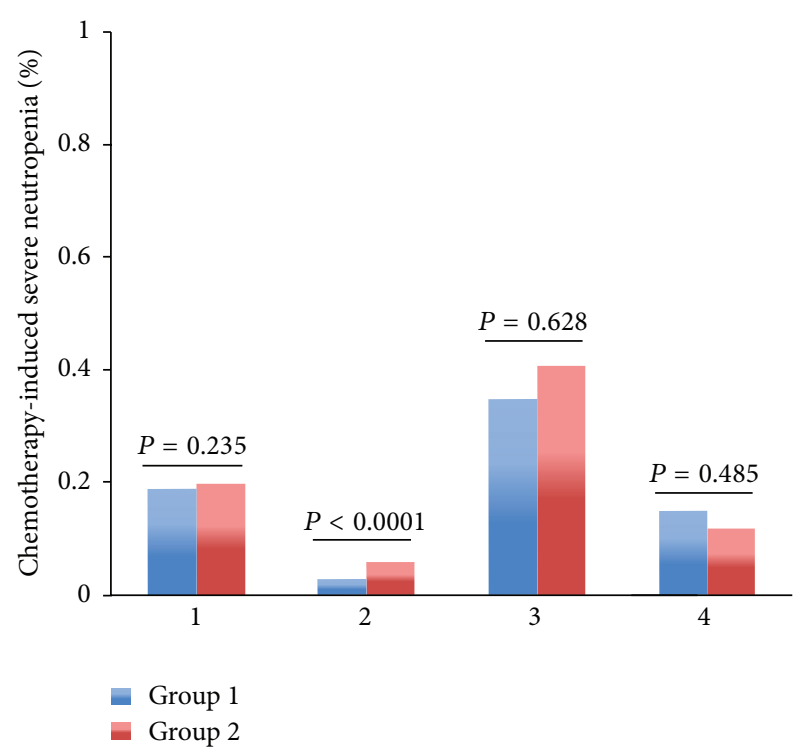

FIGURE 4: The rates of severe chemotherapy-induced neutropenia (Grades III-IV) for the entire propensity-matched patients in different chemotherapy regimens. After matching, patients in group 2 had a higher rate of severe neutropenia compared to patients in group 1 who received chemotherapy regimens 1,2 , and 3 treatment (20\% versus $19 \%, P=0.235 ; 6 \%$ versus $3 \%, P<0.0001 ; 41 \%$ versus $35 \%, P=0.628$, resp.). However, patients in group 1 had a higher rate of severe neutropenia compared to patients in group 2 who received chemotherapy regimen 4 treatment (15\% versus $12 \%, P=0.485)$. Chemotherapy. 1: the chemotherapy regimens contain AC/EC (Adriamycin or epirubicin, Cyclophosphamide), CAF/CEF (Adriamycin or epirubicin, Cyclophosphamide, and 5Fluorouracil). 2: the chemotherapy regimens contain TC (paclitaxel or docetaxel, Cyclophosphamide) and T (paclitaxel or docetaxel). 3: the chemotherapy regimens contain anthracyclines combined with paclitaxel or docetaxel: TAC/TEC (paclitaxel or docetaxel, Cyclophosphamide, and Adriamycin or epirubicin); TA/TE (paclitaxel or docetaxel, Adriamycin or epirubicin). 4: the chemotherapy regimens contain anthracyclines followed by paclitaxel or docetaxel: AC/EC (Adriamycin or epirubicin, Cyclophosphamide); CAF/CEF (Adriamycin or epirubicin, Cyclophosphamide, and 5Fluorouracil), followed with $\mathrm{T}$ or $\mathrm{TH}$ (paclitaxel or docetaxel, Herceptin).

the patients in the control group developed FN, however, in the TCM treatment group only $6 \%$ of the patients did. This result might indicate that TCM was another good choice for chemotherapy-induced myelosuppression patients, especially for patients who suffered from G-CSF intolerance or allergy. However, the effects of TCM on leukopenia, neutropenia, and FN were not exactly the same. In our study, TCM treatment showed more benefits in relieving chemotherapy-induced leukopenia and FN in most patients but just acted on chemotherapy-induced neutropenia in some patients. Among patients with regimens containing anthracyclines combined with paclitaxel or docetaxel, TCM gave no relief from leukopenia and neutropenia but provided lower rate of FN compared with control group. These data suggested that the effects of TCM on myelosuppression may not be fully in accord with G-CSF but may be achieved

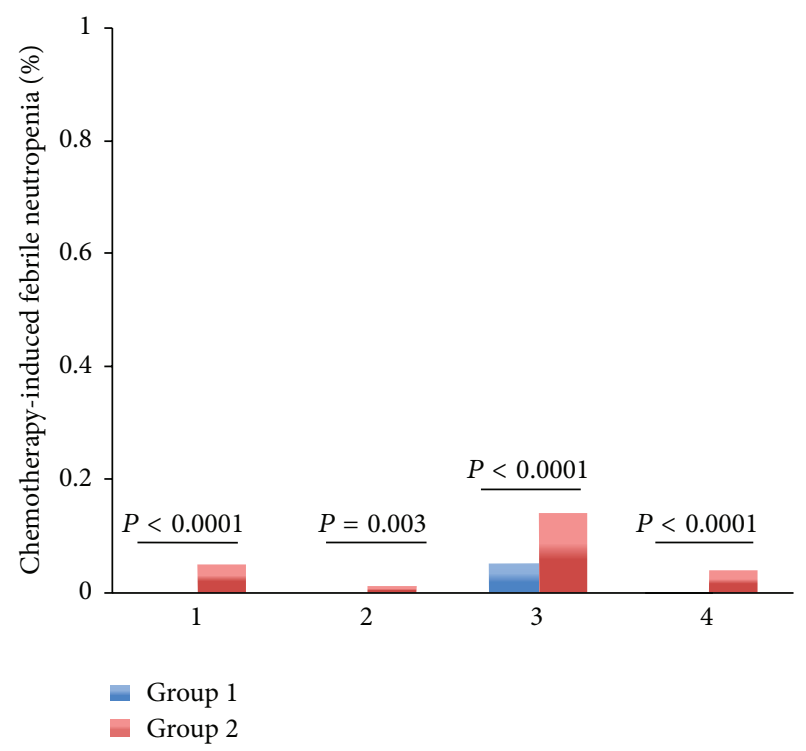

Figure 5: The rates of chemotherapy-induced FN for the entire propensity-matched patients in different chemotherapy regimens. After matching, patients in group 2 had a significantly higher rate of FN compared to patients in group 1 (5\% versus $0 \%, P<0.0001$; $1 \%$ versus $0 \%, P=0.003 ; 14 \%$ versus $5 \%, P<0.0001 ; 4 \%$ versus $0 \%$, $P<0.0001$, resp.). Chemotherapy: 1: the chemotherapy regimens contain AC/EC (Adriamycin or epirubicin, Cyclophosphamide) and CAF/CEF (Adriamycin or epirubicin, Cyclophosphamide, and 5Fluorouracil). 2: the chemotherapy regimens contain TC (paclitaxel or docetaxel, Cyclophosphamide) and $\mathrm{T}$ (paclitaxel or docetaxel). 3: the chemotherapy regimens contain anthracyclines combined with paclitaxel or docetaxel: TAC/TEC (paclitaxel or docetaxel, Cyclophosphamide, and Adriamycin or epirubicin); TA/TE (paclitaxel or docetaxel, Adriamycin or epirubicin). 4: the chemotherapy regimens contain anthracyclines followed by paclitaxel or docetaxel: AC/EC (Adriamycin or epirubicin, Cyclophosphamide); CAF/CEF (Adriamycin or epirubicin, Cyclophosphamide, and 5Fluorouracil), followed with $\mathrm{T}$ or $\mathrm{TH}$ (paclitaxel or docetaxel, Herceptin).

by increasing neutrophilic granulocytes and other immunocytes.

In previous studies, older age, lower weight, and a higher planned dose intensity of doxorubicin, epirubicin, or docetaxel were risk factors for severe neutropenia or FN [23-25]. PR status, HER-2 status, lymphovascular invasion, comorbidities, smoking status, alcohol usage, hemoglobin levels, platelet/absolute lymphocyte/absolute monocyte counts, and creatinine level had little effect. These findings were incompletely consistent with our study.

In our trial, the multivariate analysis demonstrated that chemotherapy regimens containing anthracyclines combined with paclitaxel or docetaxel received by patients were the most significant predictor for chemotherapy-induced leukopenia, neutropenia, and FN. Tumor stage and receiving preoperative chemotherapy might be associated with the risk of leukopenia. A possible explanation was that patients in later stages were more likely treated with preoperative chemotherapy and a dose intensity regimen of doxorubicin, 


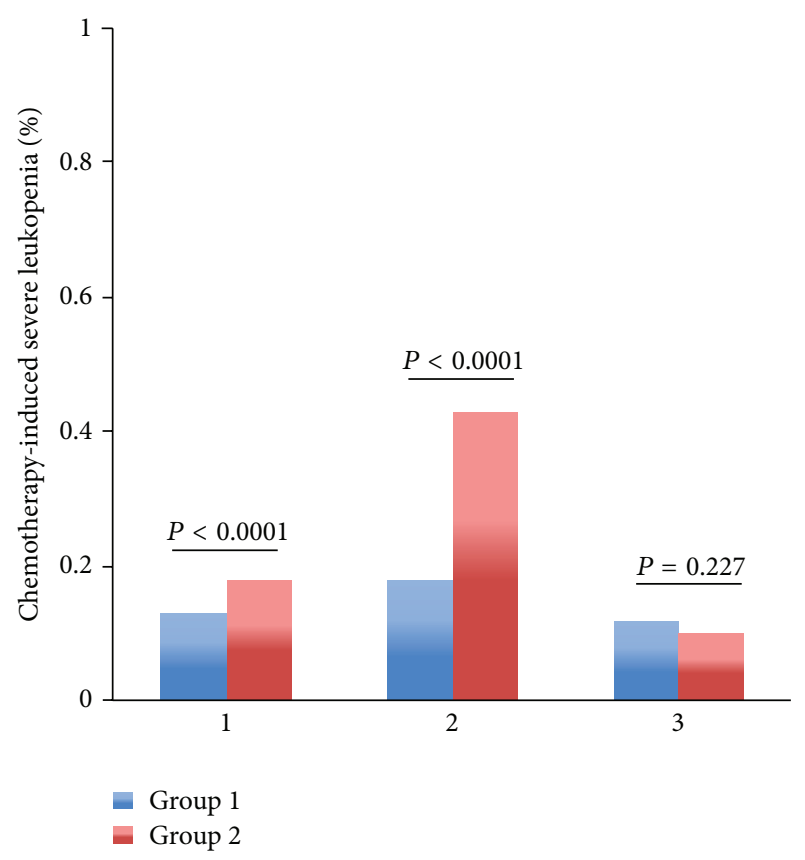

Figure 6: The rates of severe chemotherapy-induced leukopenia (Grades III-IV) for the entire propensity-matched patients in different stages. In stages 1 and 2, patients in group 2 had a significantly higher rate of severe leukopenia compared to patients in group 1 (18\% versus $13 \%, P<0.0001 ; 43 \%$ versus $18 \%, P<0.0001$, resp.). However, in stage 3, patients in group 1 had a higher rate of severe leukopenia compared to patients in group 2 (12\% versus $10 \%$, $P=0.227)$. Tumor stage: 1 indicates stage I; 2 indicates stage II; 3 indicates stages III and IV.

epirubicin, or docetaxel. These patients should be treated with G-CSF or TCM prophylaxis.

Although some previous studies had shown that factors such as age $[21,22]$ and ER positivity were associated with the risk of FN [25], our results did not support those findings. This might be because the patients in our study were very young (50 years old) and in good conditions. In our trial, the ER, PR, and HER-2 status had little effect on the risks of chemotherapy-induced myelosuppression and FN, whereas Ki-67 positivity increased the risk of leucocytes. It was possible that Ki-67 positivity correlated with the degree of malignancy and that patients would be more likely to be treated with preoperative chemotherapy and a dose intensity regimen of doxorubicin, epirubicin, or docetaxel. As to HER2 , one possible explanation was that patients with HER-2 positivity were more likely to be treated with chemotherapy regimen which contains anthracyclines followed by paclitaxel or docetaxel and Herceptin, if not the result of chance.

Multivariate analyses had confirmed the effect of TCM treatment on chemotherapy-induced myelosuppression and FN. For most Chinese patients, TCM was much more easily accessed and maintained than G-CSF. In addition, TCM was convenient to use because of its oral administration and lack of adverse events, as in our study it showed little hepatic or renal dysfunction, and it was cheap. In

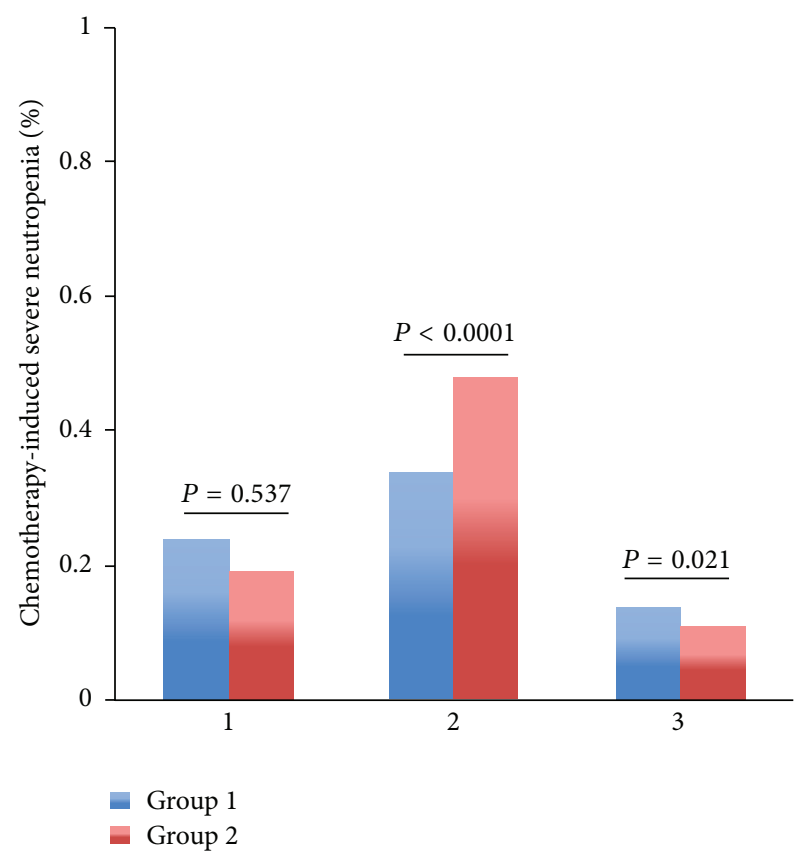

FIGURE 7: The rates of severe chemotherapy-induced neutropenia (Grades III-IV) for the entire propensity-matched patients in different stages. After matching, in stages 1 and 3, patients in group 1 had a higher rate of severe neutropenia compared to patients in group 2 (24\% versus $19 \%, P=0.537 ; 14 \%$ versus $11 \%, P=0.021$, resp.). However, in stage 2 , patients in group 2 had a significantly higher rate of severe neutropenia compared to patients in group 1 ( $48 \%$ versus $34 \%, P<0.0001$ ). Tumor stage: 1 indicates stage I; 2 indicates stage II; 3 indicates stages III and IV.

fact, TCM could be used alone or in combination with pharmacological agents, which might improve the efficacy and decrease adverse events. TCM treatment might be an optimal alternative therapy for chemotherapy-induced myelosuppression and FN patients. But our data also indicated that TCM showed little effect on patients at stage III and received regimens containing anthracyclines combined with paclitaxel or docetaxel. In our view, these patients were in serious condition, often bone marrow micrometastasis, which might damage hematopoietic function. In addition, the chemotherapy would cause severe nausea and vomiting, poor appetite, dyssomnia, and suppressed assimilation, which lead to hemopoiesis injured ultimately.

Because our study is a retrospective analysis, a randomized, controlled study is necessary to confirm our results. And some molecular biology experiments are needed to identify the molecular action of TCM on myelosuppression and FN.

\section{Conflict of Interests}

All authors declare no conflict of interests.

\section{Authors' Contribution}

Huan Tian and Wei Qin contributed equally to this study. 


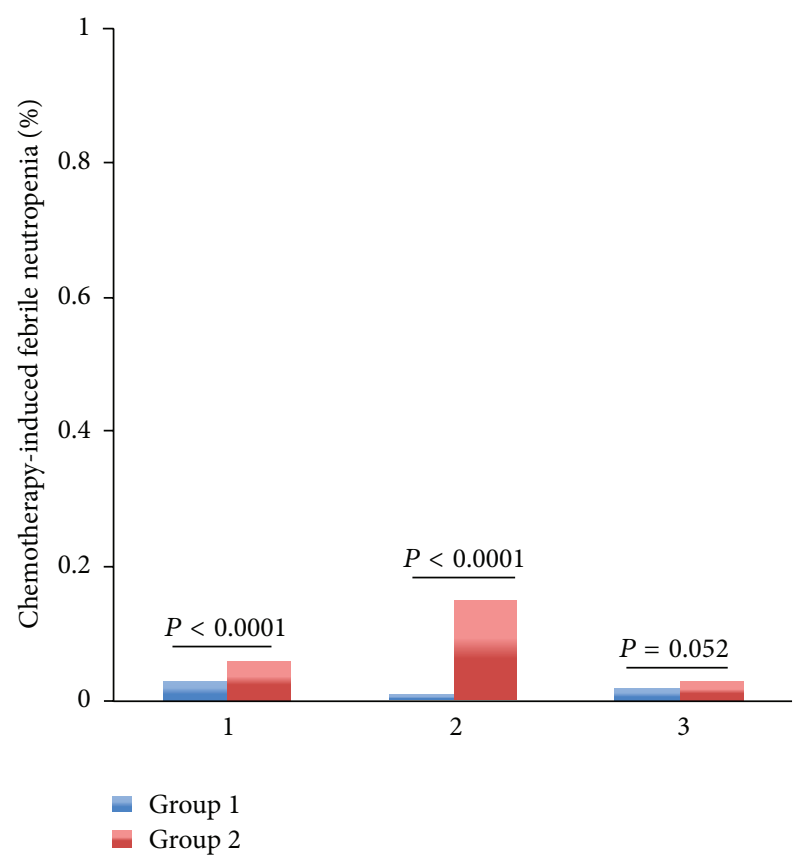

FIGURE 8: The rates of chemotherapy-induced FN for the entire propensity-matched patients in different stages. After matching, patients in group 2 had a higher rate of FN compared to patients in group 1 (6\% versus $3 \%, P<0.0001 ; 15 \%$ versus $1 \%, P<0.0001$; $3 \%$ versus $2 \%, P=0.052$, resp.). Tumor stage: 1 indicates stage I; 2 indicates stage II; 3 indicates stages III and IV.

\section{Acknowledgments}

The authors thank Dr. Jinghua Zhao, Dr. Tingting Hu, Ms. Qian Li, and Ms. Chunmei Wu from Sun Yat-sen Memorial Hospital for their help in providing much valuable data of breast cancer patients. They thank Dr. Kai Chen for his helpful discussion. This work was supported by grants from the National Natural Science Foundation of China (81172524 and 81372817).

\section{References}

[1] J. Crawford, D. C. Dale, and G. H. Lyman, "Chemotherapyinduced neutropenia: risks, consequences, and new directions for its management," Cancer, vol. 100, no. 2, pp. 228-237, 2004.

[2] A. G. Freifeld, E. J. Bow, K. A. Sepkowitz et al., "Executive summary: clinical practice guideline for the use of antimicrobial agents in neutropenic patients with cancer: 2010 update by the Infectious Diseases Society of America," Clinical Infectious Diseases, vol. 52, no. 4, pp. 427-431, 2011.

[3] A. Bosly, D. Bron, A. Van Hoof et al., "Achievement of optimal average relative dose intensity and correlation with survival in diffuse large B-cell lymphoma patients treated with CHOP," Annals of Hematology, vol. 87, no. 4, pp. 277-283, 2008.

[4] I. Chirivella, B. Bermejo, A. Insa et al., "Optimal delivery of anthracycline-based chemotherapy in the adjuvant setting improves outcome of breast cancer patients," Breast Cancer Research and Treatment, vol. 114, no. 3, pp. 479-484, 2009.

[5] R. Pettengell, H. E. Johnson, P. J. Lugtenburg et al., "Impact of febrile neutropenia on R-CHOP chemotherapy delivery and hospitalizations among patients with diffuse large B-cell lymphoma," Supportive Care in Cancer, vol. 20, no. 3, pp. 647652, 2012.

[6] L. Yi-Hsien and C. Jen-Hwey, "Use of Chinese medicine by women with breast cancer: a nationwide cross-sectional study in Taiwan," Complementary Therapies in Medicine, vol. 19, no. 3 , pp. 137-143, 2011.

[7] C.-G. Mao, Z.-Z. Tao, L.-J. Wan, J.-B. Han, Z. Chen, and B.K. Xiao, "The efficacy of traditional Chinese Medicine as an adjunctive therapy in nasopharyngeal carcinoma: a systematic review and meta-analysis," Journal of the Balkan Union of Oncology, vol. 19, no. 2, pp. 540-548, 2014.

[8] G. Gu, I. Barone, L. Gelsomino et al., "Oldenlandia diffusa extracts exert anti-proliferative and apoptotic effects on human breast cancer cells through ER $\alpha /$ Spl-mediated p53 activation," Journal of Cellular Physiology, vol. 227, no. 10, pp. 3363-3372, 2012.

[9] A. T. Perez, B. Arun, D. Tripathy et al., "A phase $1 B$ dose escalation trial of Scutellaria barbata (BZL101) for patients with metastatic breast cancer," Breast Cancer Research and Treatment, vol. 120, no. 1, pp. 111-118, 2010.

[10] A. G. Freifeld, E. J. Bow, K. A. Sepkowitz et al., "Clinical practice guideline for the use of antimicrobial agents in neutropenic patients with cancer: 2010 update by the Infectious Diseases Society of America,' Clinical Infectious Diseases, vol. 52, no. 4, pp. e56-e93, 2011.

[11] M. S. Aapro, J. Bohlius, D. A. Cameron et al., "2010 update of EORTC guidelines for the use of granulocyte-colony stimulating factor to reduce the incidence of chemotherapy-induced febrile neutropenia in adult patients with lymphoproliferative disorders and solid tumours," European Journal of Cancer, vol. 47, no. 1, pp. 8-32, 2011.

[12] I. Ross and G. R. Robert, "R: a language for data analysis and graphics," Journal of Computational and Graphical Statistics, vol. 5, no. 3, pp. 299-314, 1996.

[13] L. Xu, H. Li, Z. Xu et al., "Multi-center randomized doubleblind controlled clinical study of chemotherapy combined with or without traditional Chinese medicine on quality of life of postoperative non-small cell lung cancer patients," $B M C$ Complementary and Alternative Medicine, vol. 12, article 112, 2012.

[14] C. Zhong, H.-D. Li, D.-Y. Liu et al., "Clinical study of hepatectomy combined with Jianpi Huayu therapy for hepatocellular carcinoma," Asian Pacific Journal of Cancer Prevention, vol. 15, no. 14, pp. 5951-5957, 2014.

[15] Y.-H. Huang, J.-L. Chen, S.-H. Yang, G.-H. Liu, K.-P. Chang, and N.-M. Tsang, "Influence of Chinese medicine on weight loss and quality of life during radiotherapy in head and neck cancer," Integrative Cancer Therapies, vol. 12, no. 1, pp. 41-49, 2013.

[16] Y. Xu, A. G. Zhao, Z. Y. Li et al., "Survival benefit of traditional chinese herbal medicine (a herbal formula for invigorating spleen) for patients with advanced gastric cancer," Integrative Cancer Therapies, vol. 12, no. 5, pp. 414-422, 2013.

[17] M. Liu, H. Tan, X. Zhang et al., "Hematopoietic effects and mechanisms of Fufang Ejiao Jiang on radiotherapy and chemotherapy-induced myelosuppressed mice," Journal of Ethnopharmacology, vol. 152, no. 3, pp. 575-584, 2014.

[18] A.-B. Zhao, B. Yu, X.-L. Wu et al., "Protective effects on myelosuppression mice treated by three different classic Chinese medicine formulae," Pharmacognosy Magazine, vol. 7, no. 26, pp. 133-140, 2011. 
[19] C. Liu, J. Li, F. Y. Meng et al., "Polysaccharides from the root of Angelica sinensis promotes hematopoiesis and thrombopoiesis through the PI3K/AKT pathway," BMC Complementary and Alternative Medicine, vol. 10, article 79, 2010.

[20] L.-F. Wang, Z.-Y. Xu, C.-J. Jin et al., "Dual regulation of cell cycles by Shuanghuang Shengbai Granule in Lewis-bearing mice with chemotherapy-induced myelosuppression and its mechanism," Zhong Xi Yi Jie He Xue Bao, vol. 7, no. 5, pp. 453457, 2009.

[21] A. P. Mullard, V. Misra, P. Sumra, Z. Ali, S. M. O’Reilly, and Z. Malik, "Reducing febrile neutropenia rates in early breast cancer. Experience of two UK cancer centres," Supportive Care in Cancer, vol. 22, no. 8, pp. 2033-2037, 2014.

[22] K. Chen, X. L. Zhang, H. R. Deng et al., "Clinical predictive models for chemotherapy-induced febrile neutropenia in breast cancer patients: a validation study," PLoS ONE, vol. 9, no. 6, Article ID e96413, 2014.

[23] M. Schwenkglenks, R. Pettengell, C. Jackisch et al., "Risk factors for chemotherapy-induced neutropenia occurrence in breast cancer patients: data from the INC-EU Prospective Observational European Neutropenia Study," Supportive Care in Cancer, vol. 19, no. 4, pp. 483-490, 2011.

[24] L. Jolis, F. Carabantes, S. Pernas et al., "ncidence of chemotherapy-induced neutropenia and current practice of prophylaxis with granulocyte colony-stimulating factors in cancer patients in Spain: a prospective, observational study," European Journal of Cancer Care, vol. 22, no. 4, pp. 513-521, 2013.

[25] J. A. Chiarotto and G. Dranitsaris, "Full-dose chemotherapy in early stage breast cancer regardless of absolute neutrophil count and without G-CSF does not increase chemotherapy-induced febrile neutropenia," Supportive Care in Cancer, vol. 21, no. 10, pp. 2727-2731, 2013. 


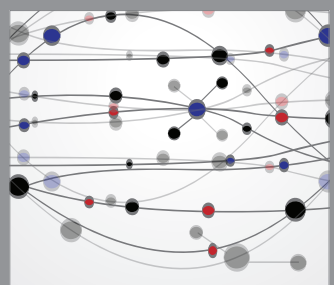

The Scientific World Journal
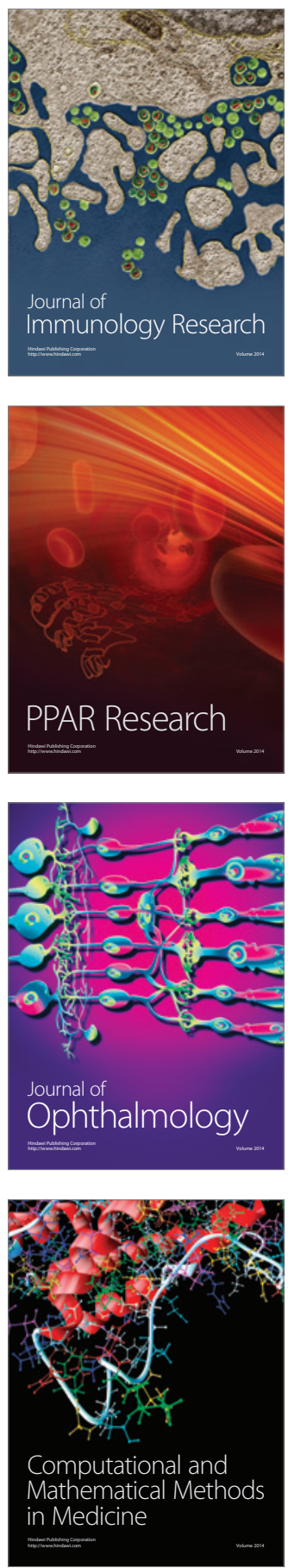

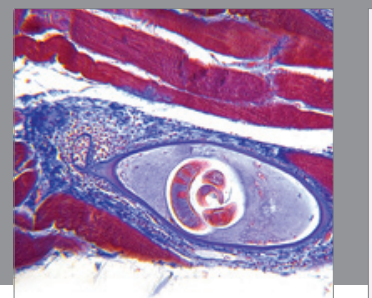

Gastroenterology

Research and Practice
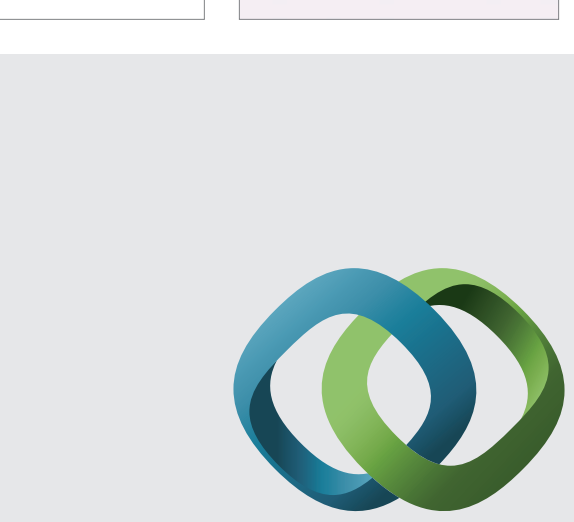

\section{Hindawi}

Submit your manuscripts at

http://www.hindawi.com
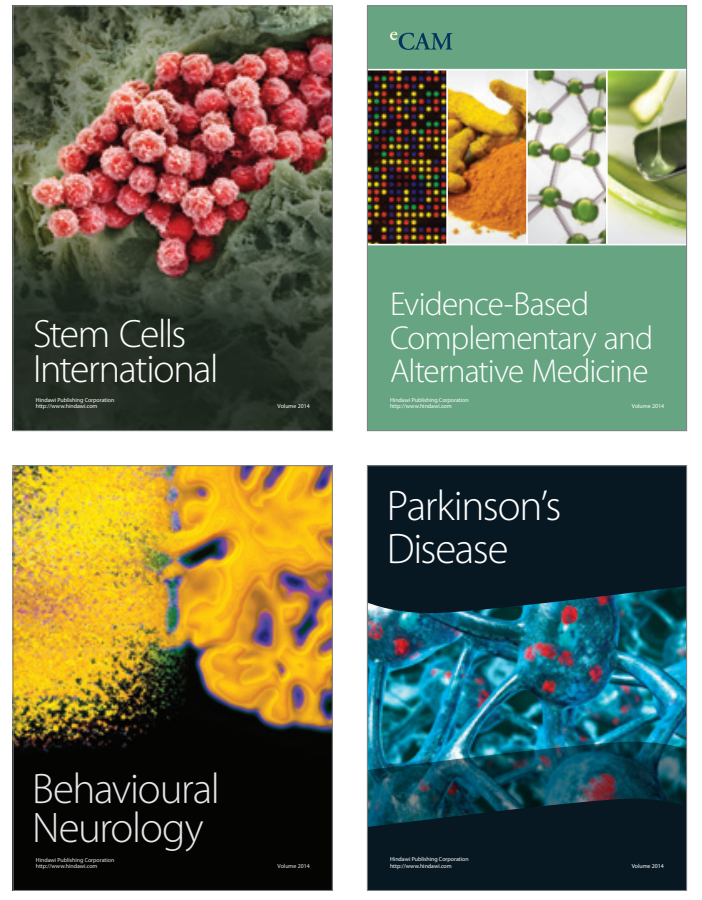
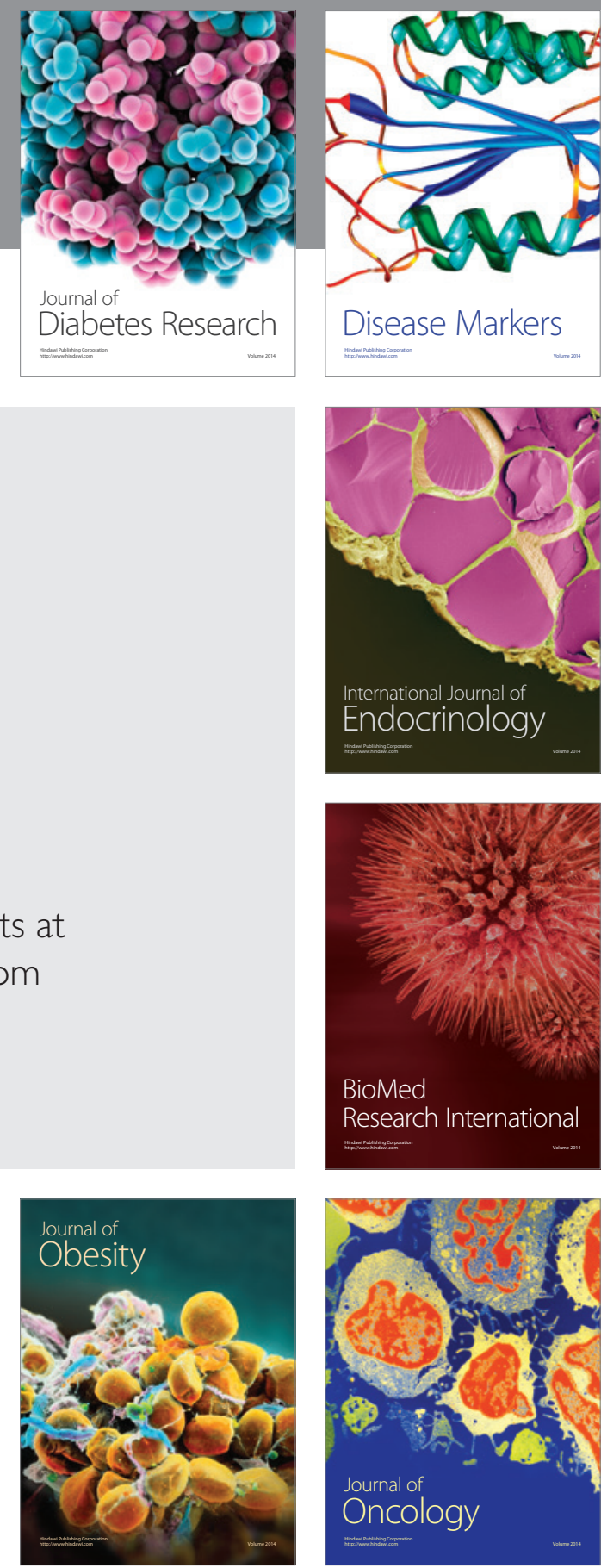

Disease Markers
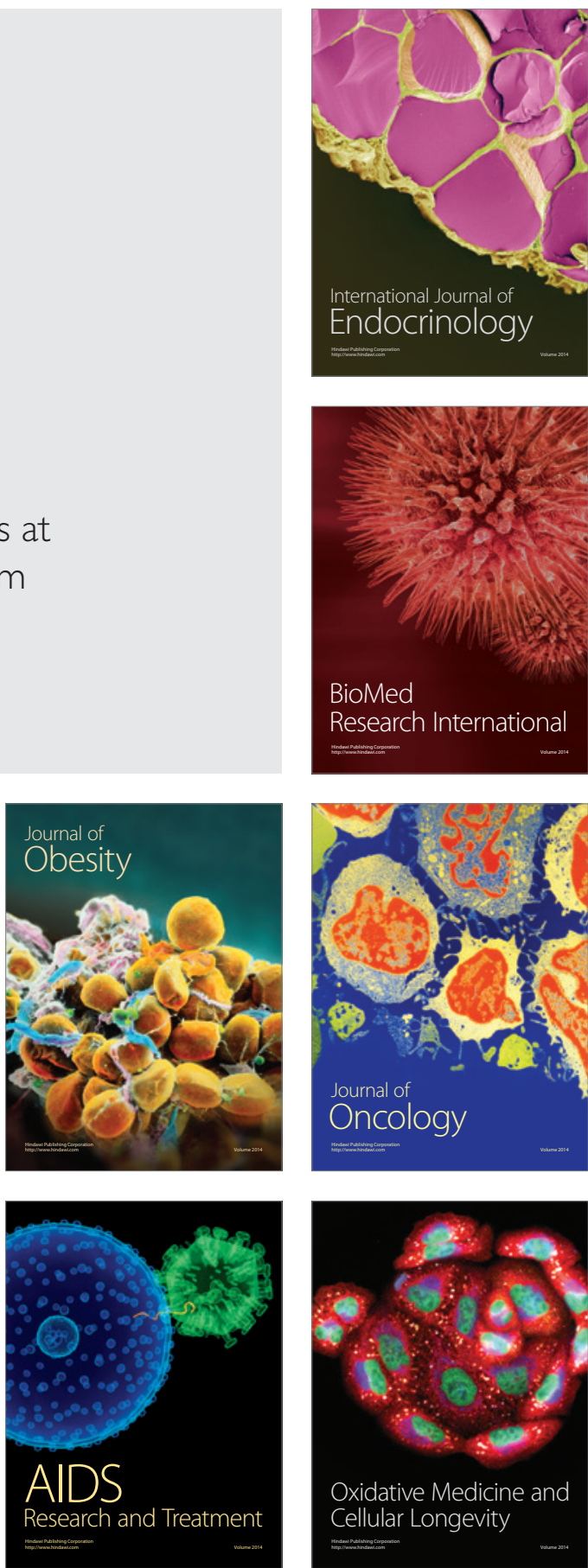